\title{
Newtonian and special-relativistic predictions for the trajectories of a low-speed scattering system
}

\author{
Boon Leong Lan ${ }^{1}$ and F. Borondo ${ }^{2}$ \\ ${ }^{1}$ School of Science, Monash University, 46150 Bandar Sunway, Selangor, Malaysia \\ ${ }^{2}$ Departamento de Quimica and Instituto Mixto de Ciencias Matematicas CSIC-UAM-UC3M-UCM, Universidad Autonoma de Madrid, \\ Cantoblanco, E-28049 Madrid, Spain
}

(Received 13 August 2010; published 7 March 2011)

\begin{abstract}
Newtonian and special-relativistic predictions, based on the same model parameters and initial conditions for the trajectory of a low-speed scattering system are compared. When the scattering is chaotic, the two predictions for the trajectory can rapidly diverge completely, not only quantitatively but also qualitatively, due to an exponentially growing separation taking place in the scattering region. In contrast, when the scattering is nonchaotic, the breakdown of agreement between predictions takes a very long time, since the difference between the predictions grows only linearly. More importantly, in the case of low-speed chaotic scattering, the rapid loss of agreement between the Newtonian and special-relativistic trajectory predictions implies that special-relativistic mechanics must be used, instead of the standard practice of using Newtonian mechanics, to correctly describe the scattering dynamics.
\end{abstract}

DOI: 10.1103/PhysRevE.83.036201

PACS number(s): 05.45.Pq

\section{INTRODUCTION}

The standard practice $[1,2]$ in the field of nonlinear dynamics and chaos when applied to physics and engineering problems is to use Newtonian mechanics, instead of specialrelativistic mechanics, to study the trajectories of a slowmoving dynamical system (slow meaning that the speed of the system is much smaller than the speed of light $c$ ) provided that gravity does not play a dynamical role. The reason for the standard practice is the conventional belief [3-5] that, in general, if the speed of the dynamical system is low, trajectories predicted by special-relativistic mechanics are well approximated by trajectories calculated with Newtonian mechanics using the same model parameters and initial conditions.

However, it was recently shown numerically for simple models of nondissipative [6,7] and dissipative [8,9] spatially bounded dynamical systems that, although the speed of the system is low, the Newtonian trajectory rapidly diverges from the special-relativistic counterpart if (i) the two trajectories are chaotic in the nondissipative case or (ii) the two trajectories are chaotic or transiently chaotic in the dissipative case. In contrast, the disagreement occurs very slowly if the trajectories are nonchaotic. Other studies on the relativistic dynamics of nonlinear systems have been done previously, but comparison with the Newtonian dynamics at low speed was not made. For example, following the work by Zaslavskii [10], which showed that a charged particle exposed to a broadband electric field in a static magnetic field acts like an electrically kicked harmonic oscillator where the existence of a stochastic web allows the particle to diffuse into the high-energy region, other authors $[11,12]$ have studied in detail the corresponding relativistic dynamics. Later Horwitz and Ashkenazy reported additional work on the relativistic kicked oscillator using the proper covariant Lorentz force [13] and taking also into account the effect of radiation on the stochastic web [14]. Matrasulov and coworkers considered the transport properties in the relativistic periodically kicked rotor [15] and the diffusive ionization of a relativistic hydrogen-like atom [16].
In this paper, we extend the comparison of Newtonian and special-relativistic trajectories to the case of low-speed scattering (spatially unbounded) system that can exhibit chaotic or irregular behavior. For reviews of nonrelativistic classical chaotic scattering see, for example, Refs. [17-21]. As a working example we consider here a model first introduced by Beeker and Eckelt [22] to study chaotic scattering in a periodically driven Hamiltonian system of one degree of freedom from the Newtonian perspective, using the associated exact two-dimensional map.

The organization of the paper is as follows. In Sec. II, we will present the details of the scattering system and derive an exact two-dimensional map for the special-relativistic dynamics. Typical results for scattering trajectories are presented and discussed in Sec. III. Finally, in Sec. IV, we summarize our conclusions and discuss the meaning and potential consequences of our findings.

\section{SCATTERING SYSTEM AND MAPS}

The scattering system that we have chosen to study consists of a particle of rest mass $m_{0}$ moving in the one-dimensional potential well [22]

$$
V(x)=-\frac{V_{0}}{\beta}\left(1+x^{2}\right)^{-\beta / 2},
$$

which is periodically turned on only for an instant of time. The potential well is characterized by two parameters $V_{0}$ and $\beta$, where $V_{0} / \beta$ determines the depth of the well and $\beta$ determines its asymptotic behavior. The special-relativistic equations of motion for the periodically driven particle are given by

$$
\begin{gathered}
\frac{d x}{d t}=\frac{p}{m_{0} \sqrt{1+\left[p /\left(m_{0} c\right)\right]^{2}}}, \\
\frac{d p}{d t}=-\frac{d V}{d x} \delta_{T}(t)=-V_{0} x\left(1+x^{2}\right)^{-(\beta+2) / 2} \delta_{T}(t),
\end{gathered}
$$


where $\delta_{T}(t)$ is a periodic series of delta functions, with period $T$, given by

$$
\delta_{T}(t)=T \sum_{j=-\infty}^{\infty} \delta(j T-t) .
$$

It is easily seen from Eq. (2) that low particle momentum, $p \ll m_{0} c$, implies low particle speed, $v \ll c$.

The practical reason for using periodically driven systems as dynamical models is that the associated equations of motion can be easily converted into discrete maps, where the numerical computations become much easier and accurate. In our case the derivation of the special-relativistic map is as follows. Across the $n$th kick, that is, from time $t=n T^{-}$(just before the $n$th kick) to $t=n T^{+}$(just after the $n$th kick), the position does not change:

$$
x\left(n T^{+}\right)=x\left(n T^{-}\right) .
$$

However, the momentum changes as a result of the kick:

$$
\frac{d p}{d t}=-V_{0} x\left(n T^{-}\right)\left[1+x^{2}\left(n T^{-}\right)\right]^{-(\beta+2) / 2} \delta_{T}(t) .
$$

Integrating Eq. (6) from $t=n T^{-}$to $t=n T^{+}$yields

$$
p\left(n T^{+}\right)=p\left(n T^{-}\right)-V_{0} T x\left(n T^{-}\right)\left[1+x^{2}\left(n T^{-}\right)\right]^{-(\beta+2) / 2} .
$$

In between the $n$th kick and $(n+1)$-th kick, that is, from $t=n T^{+}$(just after the $n$th kick) to $t=(n+1) T^{-}$(just before the $(n+1)$-th kick), the momentum does not change:

$$
p\left((n+1) T^{-}\right)=p\left(n T^{+}\right)
$$

since the particle evolves freely. The position changes according to

$$
\frac{d x}{d t}=\frac{p\left(n T^{+}\right)}{m_{0} \sqrt{1+\left[p\left(n T^{+}\right) /\left(m_{0} c\right)\right]^{2}}} .
$$

Integrating Eq. (9) from $t=n T^{+}$to $t=(n+1) T^{-}$gives

$$
x\left[(n+1) T^{-}\right]=x\left(n T^{+}\right)+\frac{T p\left(n T^{+}\right)}{m_{0} \sqrt{1+\left[p\left(n T^{+}\right) /\left(m_{0} c\right)\right]^{2}}} .
$$

Using Eqs. (5), (8), (7), and (10) can finally be written as

$$
\begin{aligned}
& p_{n+1}=p_{n}-V_{0} T x_{n}\left(1+x_{n}^{2}\right)^{-(\beta+2) / 2}, \\
& x_{n+1}=x_{n}+\frac{T p_{n+1}}{m_{0} \sqrt{1+\left[p_{n+1} /\left(m_{0} c\right)\right]^{2}}} .
\end{aligned}
$$

The special-relativistic expressions given by Eqs. (11) and (12) map the position and momentum of the particle from just before the $n$th kick to just before the $(n+1)$-th kick.

The corresponding Newtonian equations of motion for the particle are given by Eqs. (2) and (3) without the square-root term in Eq. (2). A similar derivation to that performed above leads to the associated Newtonian map [22]:

$$
\begin{gathered}
p_{n+1}=p_{n}-V_{0} \operatorname{Tx}_{n}\left(1+x_{n}^{2}\right)^{-(\beta+2) / 2}, \\
x_{n+1}=x_{n}+\frac{T}{m_{0}} p_{n+1} .
\end{gathered}
$$

Beeker and Eckelt [22] chose $m_{0}=1$ and $T=1$ for the rest mass of the particle and the kicking period, respectively, in their study of the Newtonian map [Eqs. (13) and (14)]. They found that for $0<V_{0}<4$ the scattering is nonchaotic, meaning that the scattering function varies smoothly or regularly. However, for values of the potential parameters $V_{0}=8$ and $\beta=4$, they found that the scattering is chaotic. In this case, the scattering function (see Fig. 2 in Ref. [22]) varies irregularly in certain intervals, and the irregular and regular intervals intertwine down to all scales. Moreover, for these potential parameter values, the chaotic scattering is Ref. [22] "fully developed" [23], in the sense that all periodic orbits are unstable and there are no Kolmogorov-Arnold-Moser islands in the scattering region.

\section{RESULTS}

Following Beeker and Eckelt [22], we also use $m_{0}=1$ and $T=1$ for the rest mass of the particle and the kicking period in our calculations. In addition, we take $10^{5}$ as the value for the speed of light $c$ and $\beta=4$. Recall that, from the Newtonian perspective, the scattering is [22] nonchaotic for $V_{0}=2$, but chaotic for $V_{0}=8$. We found that the scattering is also correspondingly nonchaotic and chaotic when the specialrelativistic dynamics at low speed is considered.

In this section we present and discuss typical results obtained from our calculations when comparing the low-speed scattering trajectories predicted by the special-relativistic map [Eqs. (11) and (12)] and the Newtonian map [Eqs. (13) and (14)] using the same initial conditions, and considering cases where the scattering is nonchaotic $\left(V_{0}=2\right.$ and $\left.\beta=4\right)$ and chaotic $\left(V_{0}=8\right.$ and $\left.\beta=4\right)$.

Figure 1 shows the potential well and the corresponding force for $V_{0}=8$ and $\beta=4$. The force decays rapidly to its asymptotic zero value. The behavior of the force is similar for $V_{0}=2$ and $\beta=4$. We have verified that the trajectories we have studied are numerically accurate by comparing double and quadruple-precision calculations. In all cases, the initial position of the particle was well outside the scattering region.

The first example is from the nonchaotic scattering case. The Newtonian and special-relativistic trajectories, starting from the same initial position, $x_{0}=-20$, and initial momentum, $p_{0}=1.2497$, are plotted in Fig. 2. The magnitude of the special-relativistic momentum remains close to 1 , which

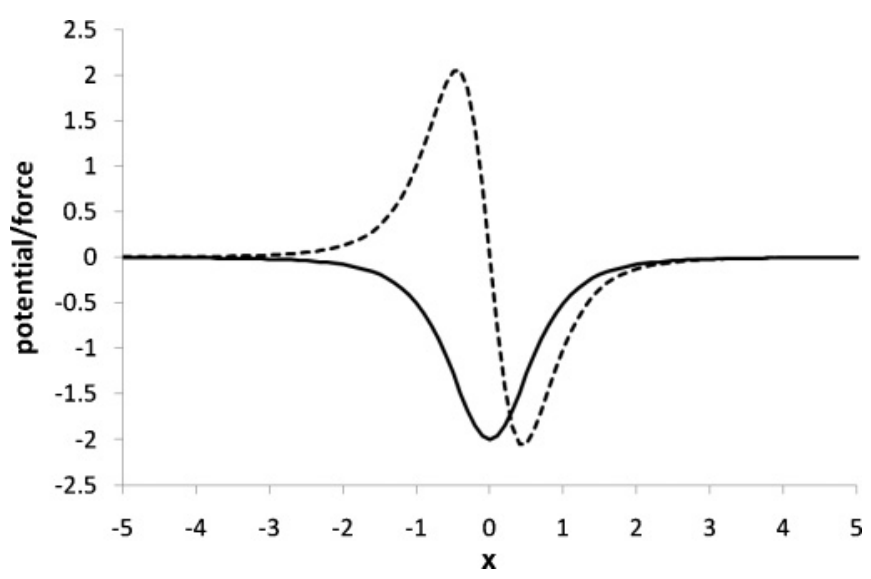

FIG. 1. Scattering potential (solid line) of Eq. (1) and the corresponding force (dotted line) for $V_{0}=8$ and $\beta=4$. 

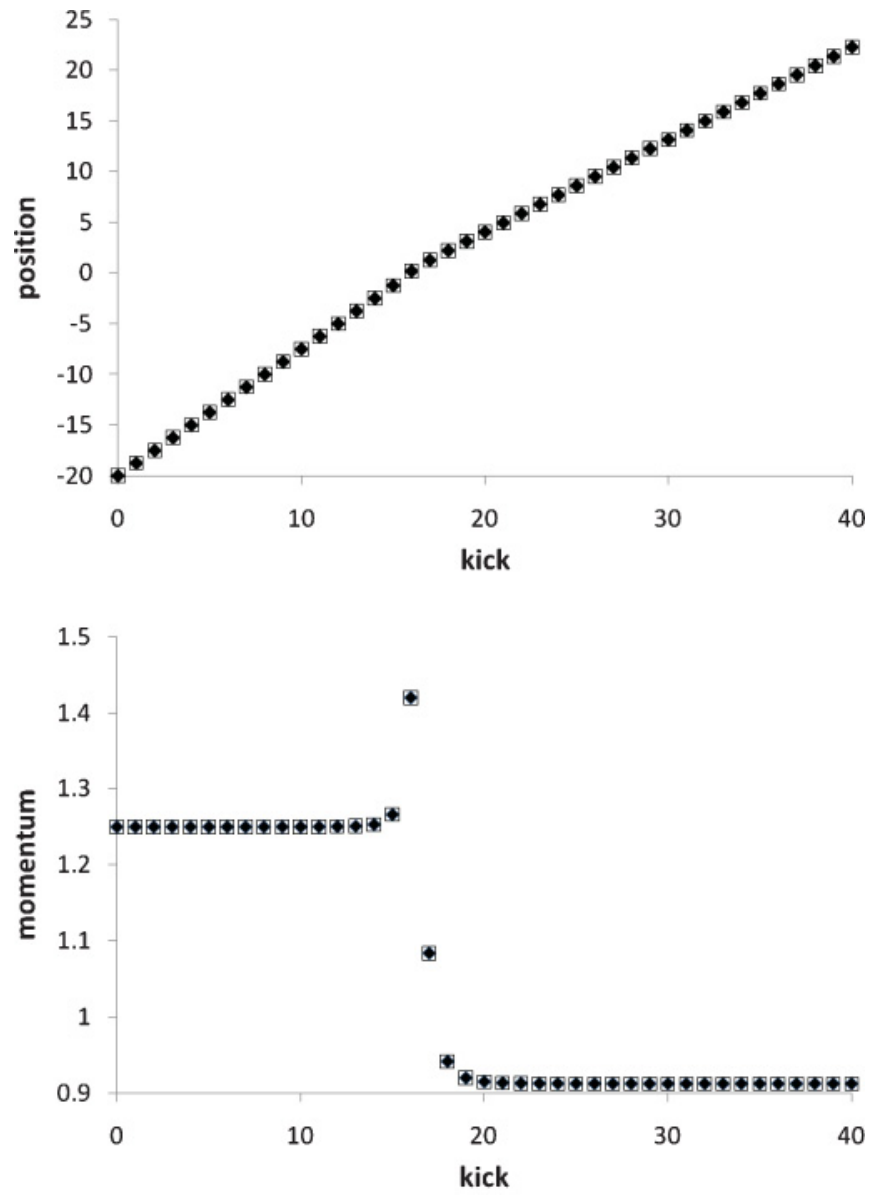

FIG. 2. Newtonian and special-relativistic predictions for the nonchaotic scattering case: position (top) and momentum (bottom). Newtonian and special-relativistic values are plotted with squares and diamonds, respectively.

is $10^{-5} c$. Expression (2) thus implies that the speed of the particle is $10^{-5} c$; that is, the speed of the particle is only $0.001 \%$ the speed of light. As can be seen in Fig. 2, our calculation shows that both theories predict that the particle is transmitted, and the trajectory obtained from the specialrelativistic theory is well approximated by the Newtonian trajectory, as conventionally expected for low-speed scattering.

The next example is, on the other hand, taken from the chaotic scattering case. The initial position $x_{0}$ is -20 , and the initial momentum $p_{0}$ is 1.2497 for both the Newtonian and special-relativistic trajectories. The magnitude of the specialrelativistic momentum remains close to 1 , again meaning that the speed of the particle is only $0.001 \%$ the speed of light. The obtained results, shown in Fig. 3, demonstrate that, contrary to the usual expectations, the special-relativistic trajectory is well approximated by the Newtonian trajectory only for a short period of time, even in this case where the particle speed remains low. Actually, after 35 kicks, the two trajectories look completely different. Moreover, it should be remarked that in this example the predictions of the two theories are not only quantitatively different but also differ qualitatively: While the particle is reflected according to special-relativistic mechanics, it is transmitted in the same circumstances according to Newtonian mechanics. In
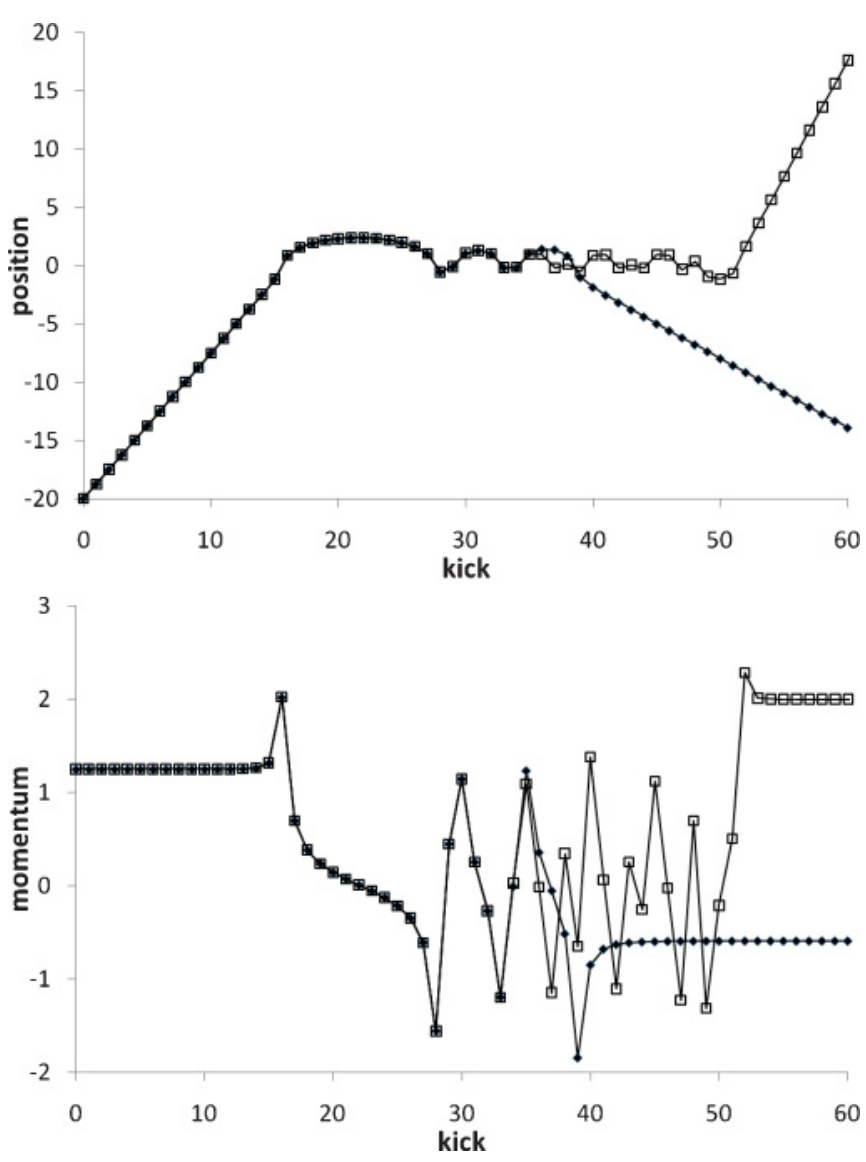

FIG. 3. Newtonian and special-relativistic predictions for the first example of the chaotic scattering case: position (top) and momentum (bottom). Newtonian and special-relativistic values are plotted with squares and diamonds, respectively.

addition, Newtonian mechanics predicts that the particle remains in the scattering region significantly longer-it exits after 53 kicks - than in the special relativistic case, for which it leaves the interaction region much earlier, after only 43 kicks.

The magnitude of the difference between the Newtonian and special-relativistic predictions is plotted versus the kick numbers in Figs. 4 and 5, for the nonchaotic and chaotic scattering cases, respectively. For both cases, the asymptotic, either incoming or outgoing, momentum difference is easily understood. The asymptotic Newtonian momentum is constant, and so is the asymptotic special-relativistic momentum (see Figs. 2 and 3). Therefore, the asymptotic momentum difference is also a constant (see Figs. 4 and 5). Notice that the incoming asymptotic momentum difference is not shown in Fig. 5 because it has a zero value.

For both cases, the asymptotic (again either incoming or outgoing) difference in the positions is also easily understood. The asymptotic position difference varies linearly with time (see Figs. 4 and 5) since the asymptotic Newtonian position and the asymptotic special-relativistic position both vary linearly with time (see Figs. 2 and 3). The linear behavior of the asymptotic position difference appears curved in Fig. 5, where the natural log of the position difference is plotted versus kick.

However, Figs. 4 and 5 show that the intermediate time variation of the position and momentum differences in the 

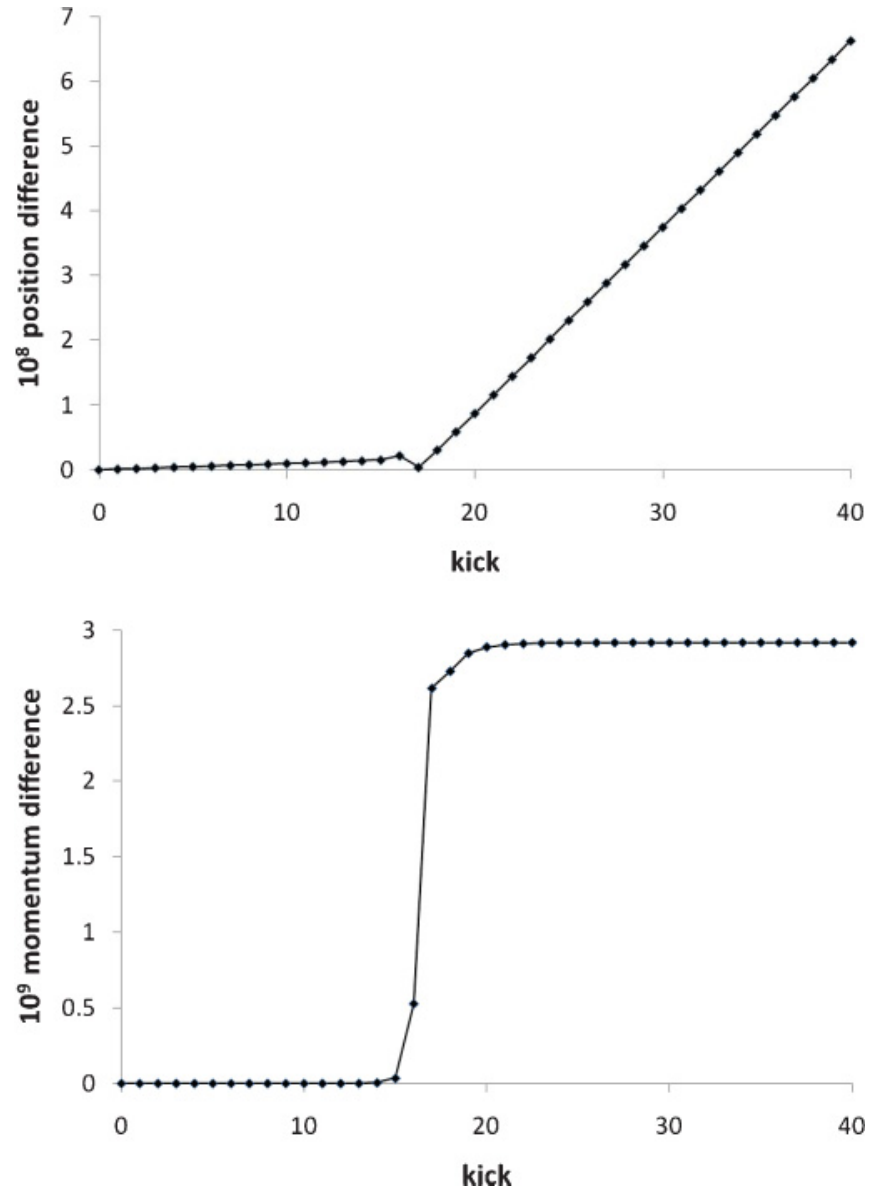

FIG. 4. Magnitude of the difference between the Newtonian and special-relativistic predictions for the nonchaotic scattering case of Fig. 2: position difference (top) and momentum difference (bottom).

scattering region are different for the two considered cases. In the nonchaotic scattering case, the position and momentum differences grow linearly with time (between kicks 14 and 19 in Fig. 4). In sharp contrast, in the irregular or chaotic scattering case, the position and momentum differences grow exponentially with time (between kicks 14 and 42 in Fig. 5). This exponential growth of the difference between trajectories leads to a complete disagreement of the two trajectory predictions after 35 kicks, as shown in Fig. 3.

In the nonchaotic scattering case, the continued slow linear growth of the position difference after the intermediate stage will eventually cause the two trajectory predictions to disagree completely, but that will take place only after a very long time.

The breakdown of agreement between the Newtonian and special-relativistic trajectory predictions in the two cases above can be further understood in the following way. Since $p \ll m_{0} c$, the factor $1 / \sqrt{1+\left[p_{n+1} /\left(m_{0} c\right)\right]^{2}}$ in Eq. (12) of the specialrelativistic map is approximately $1-1 / 2\left[p_{n+1} /\left(m_{0} c\right)\right]^{2}$, and therefore the special-relativistic map is approximately

$$
\begin{aligned}
& p_{n+1}=p_{n}-V_{0} T x_{n}\left(1+x_{n}^{2}\right)^{-(\beta+2) / 2}, \\
& x_{n+1}=x_{n}+\frac{T}{m_{0}} p_{n+1}-\frac{T}{2 m_{0}^{3} c^{2}} p_{n+1}^{2} .
\end{aligned}
$$
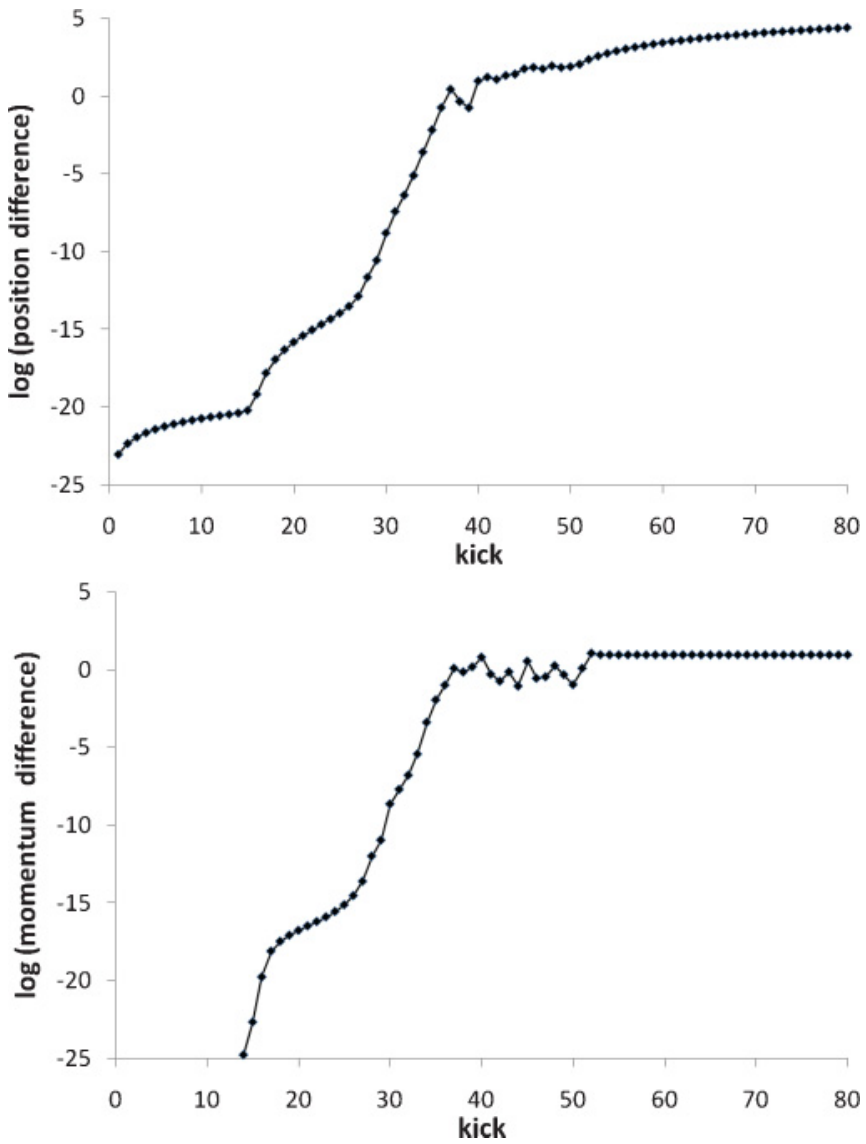

FIG. 5. Natural log of the magnitude of the difference between the Newtonian and special-relativistic predictions versus kick for the first example of the chaotic scattering case of Fig. 3: position difference (top) and momentum difference (bottom).

The last term involving $1 / c^{2}$ in Eq. (16) of the approximate special-relativistic map above can be interpreted as a small perturbation to the Newtonian map [Eqs. (13) and (14)]. For both the nonchaotic and chaotic scattering cases, the trajectories calculated using the approximate special-relativistic map above are close to the ones calculated using the exact special-relativistic map [Eqs. (11) and (12)]; Figs. 2 through 5 would not change if the approximate special-relativistic results were used in the plots. The breakdown of agreement between the Newtonian and special-relativistic trajectory predictions is therefore essentially due to the small relativistic perturbation to the Newtonian map.

We have performed calculations for other initial conditions in the chaotic scattering case, and, in general, the trajectory difference also grows exponentially in the intermediate stage. However, for some particular initial conditions, the intermediate exponential growth regime is not long enough to cause a breakdown of agreement between the two trajectory predictions. Figures 6 and 7 illustrate this scenario for the case where the initial conditions are $x_{0}=-20.494518684484$ and $p_{0}=1.2806$. However, similar to what happened in the nonchaotic scattering case, the linear growth of the position difference, after the intermediate stage between kicks 14 and 20 , eventually causes the two trajectory predictions to be completely different. 

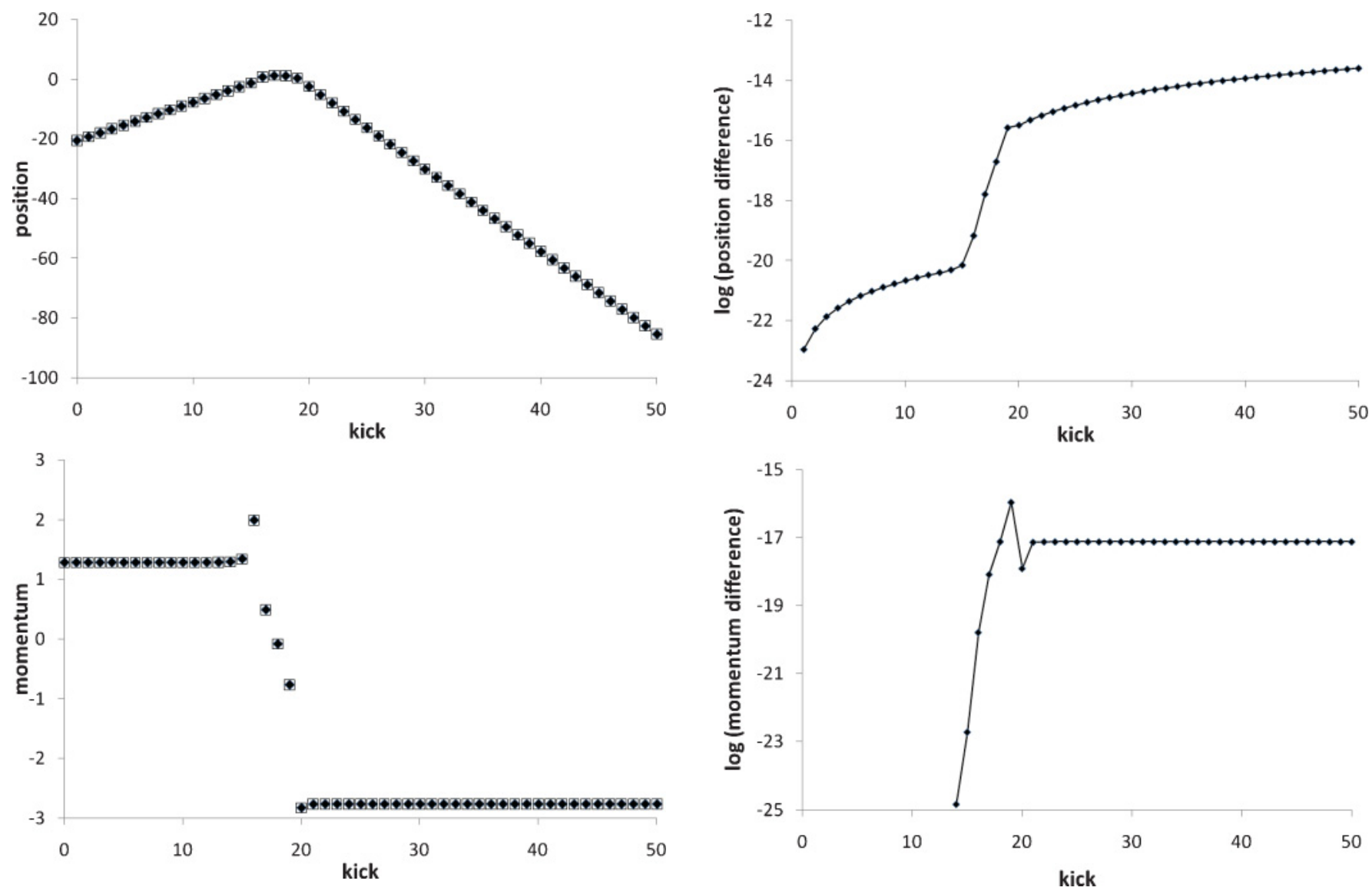

FIG. 6. Newtonian and special-relativistic predictions for the second example of the chaotic scattering case: position (top) and momentum (bottom). Newtonian and special-relativistic values are plotted with squares and diamonds, respectively.

\section{SUMMARY AND CONCLUSIONS}

We have shown that the Newtonian and special-relativistic trajectory predictions from the same initial conditions for lowspeed scattering processes can rapidly disagree completely, not only quantitatively but also qualitatively, in the case where the scattering dynamics is chaotic or irregular. This rapid breakdown of agreement is due to an exponential growth of the difference between the two trajectories taking place in the scattering or interaction region. If the exponential growth stage is not long enough to lead to a complete disagreement between the two trajectories, the subsequent linear growth of the position difference will eventually do so. In the nonchaotic scattering case, the trajectory difference grows only linearly, and therefore the breakdown of agreement between the two trajectory predictions does not occur rapidly as usually happens in the case of chaotic scattering.

Our results may have far-reaching consequences. The rapid breakdown of agreement between the Newtonian and specialrelativistic trajectories predicted for a low-speed scattering

FIG. 7. Natural log of the magnitude of the difference between the Newtonian and special-relativistic predictions versus kick for the second example of the chaotic scattering case of Fig. 6: position difference (top) and momentum difference (bottom).

system offers a new possibility for testing special relativity. Which of the two different trajectory predictions is empirically correct? This issue is especially timely, since recent tests [24-26] of the Lorentz invariance postulate of special relativity using ultrahigh-precision experiments and astrophysical observations continue to show that Lorentz invariance is not violated. Special relativistic mechanics is Lorentz invariant, but Newtonian mechanics is not. Therefore the trajectory predicted by special relativistic mechanics should be empirically correct. This means that, contrary to standard practice, special relativistic mechanics must be used, instead of Newtonian mechanics, to correctly study low-speed chaotic scattering.

\section{ACKNOWLEDGMENTS}

Support from MICINN-Spain under contract numbers MTM2009-14621 and i-MATH CSD2006-32, Centro de Estudios de America Latina y Asia (UAM)-Banco de Santander, and Fundamental Research Grant Scheme FRGS/2/2010/ST/MUSM/ 02/1 are gratefully acknowledged.

[1] F. C. Moon, Chaotic and Fractal Dynamics: An Introduction for Applied Scientists and Engineers (Wiley-Interscience, New York, 1992).
[2] S. H. Strogatz, Nonlinear Dynamics and Chaos: With Applications to Physics, Biology, Chemistry and Engineering (Perseus Books, Cambridge, MA, 2001). 
[3] A. P. French, Special Relativity (Thomas Nelson \& Sons, Cambridge, 1968), p. 167.

[4] W. D. McComb, Dynamics and Relativity (Oxford University Press, Oxford, 1999), preface.

[5] J. B. Hartle, Gravity: An Introduction to Einstein's General Relativity (Addison-Wesley, San Francisco, 2003), p. 88.

[6] B. L. Lan, Chaos 16, 033107 (2006).

[7] B. L. Lan, Chaos, Solitons \& Fractals 42, 534 (2009).

[8] B. L. Lan, in Topics on Chaotic Systems: Selected Papers from Chaos 2008 International Conference, edited by C. H. Skiadas, I. Dimotikalis, and C. Skiadas (World Scientific, 2009), p. 199.

[9] B. L. Lan and H. Y. Cheng, Commun. Nonlinear Sci. Numer. Simulat. 15, 2497 (2009).

[10] G. M. Zaslavskii, Hamiltonian Chaos and Fractional Dynamics (Oxford University Press, Oxford, 2005).

[11] D. W. Longcope and R. N. Sudan, Phys. Rev. Lett. 59, 1500 (1987).

[12] H. Karimabadi and V. Angelopoulos, Phys. Rev. Lett. 62, 2342 (1989).
[13] L. P. Horwitz and Y. Ashkenazy, Discrete Dyn. Nat. Soc. 4, 77 (2000).

[14] L. P. Horwitz and Y. Ashkenazy, Discrete Dyn. Nat. Soc. 4, 283 (2000).

[15] D. U. Matrasulov, G. M. Milibaeva, U. R. Salomov, and B. Sundaram, Phys. Rev. E 72, 016213 (2005).

[16] D. U. Matrasulov, Phys. Rev. A 60, 700 (1999).

[17] B. Eckhardt, Physica D 33, 89 (1988).

[18] E. Ott and T. Tel, Chaos 3, 417 (1993).

[19] U. Smilansky, in Les Houches, Session LII 1989, Chaos and Quantum Physics, edited by M.-J. Giannoni, A. Voros, and J. Zinn-Justin (Elsevier, Amsterdam, 1992), p. 371.

[20] Y. C. Lai and T. Tel, Transient Chaos (Springer, New York, 2010).

[21] B. Rucker and C. Jung, J. Phys. A 27, 55 (1994).

[22] A. Beeker and P. Eckelt, Chaos 3, 487 (1993).

[23] S. Bleher, E. Ott, and C. Grebogi, Phys. Rev. Lett. 63, 919 (1989).

[24] M. Pospelov and M. Romalis, Physics Today 57, 40 (2004).

[25] P. Ball, Nature (London) 427, 482 (2004).

[26] A. Cho, Science 307, 866 (2005). 